\title{
El efecto Mateo en niños con Dificultades Específicas de Aprendizaje de las Matemáticas
}

\author{
Margarita Blanco Pérez ${ }^{1}$, Vicente Bermejo²
}

\author{
${ }^{1}$ Dpto. de Psicología, Facultad de Educación y Trabajo social, Universidad de Valladolid, \\ ${ }^{2}$ Dpto. de Psicología Evolutiva y de la Educación, Facultad de Psicología, Universidad Complutense de Madrid.
}

Disponible online 22 diciembre de 2009

\begin{abstract}
En el presente trabajo se intentó determinar si la presencia de Dificultades Específicas de Aprendizaje en Matemáticas (DAM) se asociaba con la caída de las puntuaciones en las pruebas psicométricas empleadas para valorar el Cociente Intelectual (CI) que con el paso de los cursos se observa en estos niños, tal como sostienen algunos autores con las Dificultades en Lectura y Matemáticas. Además, se evaluó si las diferencias en CI se relacionaban con rendimientos matemáticos igualmente diferentes. Para ello se comparó el CI de 12 niños con DAM escolarizados en $1^{\circ}$ de Educación Primaria con el de 20 niños con DAM de $3^{\circ}$ de Educación Primaria. Por otro lado, se contrastó el CI de estos niños DAM con el de dos muestras control sin DAM de las mismas cohortes. Entre otros resultados se observó que el CI de los niños DAM de mayor edad ( $3^{\circ}$ curso) era significativamente más bajo que el de los niños más jóvenes, mientras que no existían diferencias entre los niños sin DAM. Además, la presencia de un CI alto o bajo no suponía siempre un rendimiento matemático igualmente alto o bajo en los escolares.
\end{abstract}

Palabras clave: Dificultades Específicas de Aprendizaje en Matemáticas (DAM), Discalculia, Inteligencia (CI), Efecto Mateo.

Previous research suggests that Learning Difficulties in Reading and Mathematics are highly related. This work tried to test in children if the presence of specific Learning Difficulties in Mathematics (LDM) was related to poor results in Intellectual Quotient (IQ) tests. Also, it is tested if differences in IQ are related to different mathematical performances. Therefore, the IQs of 12 LDM children enrolled in the first year of Primary School were compared with the IQs of 20 LDM children enrolled in the third year of Primary School. The IQs of LDM children were compared with the IQs of two non-LDM groups of the same cohorts. Among other results, it was observed that the IQs of the oldest LDM children were significantly lower than these of the youngest children, while no differences were found among non-LDM children. Furthermore, the presence of a high or low IQ did not always imply an equally high or low mathematical performance in the scholars.

Keywords: Specific Learning Difficulties in Mathematics (LDM), Dyscalculia, Intelligence (CI), Matthew Effect.

Correspondencia: Vicente Bermejo. Departamento de Psicología Evolutiva y de la Educación, Facultad de Psicología, Universidad Complutense, Campus de Somosaguas, 28223-Madrid. Teléfono: 9139430 86. E-mail: bermejo@psi.ucm.es 
Aunque no existe una definición rigurosa y universal de las "Dificultades específicas de Aprendizaje" (DA), la mayoría de ellas suelen basarse en tres criterios esenciales: especificidad, discrepancia y exclusión. El primero de ellos supone que las Dificultades de Aprendizaje están limitadas a un número restringido de dominios académicos y cognitivos. El segundo determina que los rendimientos no miden el potencial del alumno. Y por último, el tercero postula que las Dificultades de Aprendizaje se distinguen de otras condiciones de hándicap o desventaja (Brody y Mills, 1993).

Todos y cada uno de estos criterios han recibido diferentes críticas. El criterio de exclusión se basa en la asunción de que las DA son debidas a causas internas al individuo, a una disfunción o lesión en el sistema nervioso central (Keogh, 1990), no a factores externos. Además, estas dificultades deben ser primarias, es decir, no debidas a otras situaciones de discapacidad. Para algunos autores (Lyon et al., 2001; Siegel, 1990) la aplicación de este criterio puede perjudicar a los alumnos procedentes de entornos deprivados, al quitarles el apoyo por parte de profesores especialistas.

El criterio de especificidad y el de discrepancia están íntimamente ligados. En un plano operativo estos dos criterios suponen que los niños presentan un CI dentro de la normalidad $\mathrm{y}$, en algunos casos, un CI que discrepa de forma significativa del rendimiento en el área de su dificultad.

Según algunos autores (Keogh, 1990; Stanovich, 1986), el criterio de especificidad no debería estar sujeto a críticas, ya que su rechazo dejaría vacío de contenido el concepto de "dificultades específicas de aprendizaje". Por tanto, no negamos la necesidad de contar con este criterio, aunque consideramos necesario profundizar en su aplicación operativa, ya que la medición del CI se asienta en una serie de supuestos discutibles. Por ejemplo, que los tests de inteligencia miden capacidad intelectual, que los factores causales de las Dificultades Específicas de Aprendizaje no afectan al CI, que el CI predice el rendimiento y que los sujetos con bajo rendimiento y bajo CI son distintos de los sujetos de bajo rendimiento y mayor CI (García y Jiménez, 2000). Sin embargo, como muestran algunas investigaciones, la mayoría de los tests de CI miden conocimientos y/o habilidades en las que los niños presentan dificultades (ej.: resolución de problemas matemáticos) y no parece lógico pensar que un niño que tiene una memoria deficiente, dificultades en lenguaje o en habilidades motoras finas sea menos inteligente (Siegel, 1999). Por otro lado, es difícil cumplir el criterio de especificidad en todos los niños, pues las DA en diversas áreas pueden ir asociadas, por ejemplo, en lectura y matemáticas, pues hay aspectos cognitivos comunes a las dos habilidades, como son la memoria a corto plazo, la manipulación de símbolos gráficos, el procesamiento simbólico, la atención o la concentración (Siegel, 1990).

Diversos autores han mostrando que las DA pueden afectar al rendimiento en las pruebas de CI. Éste es el caso del estudio de Klinge, Rennick, Lennox y Hart (1977) en el que estudiaron a 30 niños que habían sido calificados por los centros educativos como con DA y los compararon con otro grupo emparejado en edad, género, raza, estatus social y comunidad geográfica. A pesar del emparejamiento, los niños con DA tenían una media de CI de 94 frente a la del grupo control que era de 103. Por su parte, Torgesen y Dice (1980) llevaron a cabo una revisión de la bibliografía científica y encontraron que la media de CI de los niños con DA era inferior en 6 puntos a la de los grupos control.

Stanovich (1986) da diversas explicaciones a estos hallazgos, resaltando que la especificidad sólo se puede valorar en los primeros años de escolaridad, pues con el paso de los años los niños empiezan a presentar problemas en más áreas debido a la transferencia motivacional, conductual y cognitiva producida por un fallo en una habilidad académica fundamental. Este autor denomina esta relación como el "efecto Mateo" ("the rich get richer and the poor get poorer"), de modo que los buenos lectores aprenden más vocabulario, lo que lleva a un aumento de su comprensión lectora y a que se conviertan en lectores más hábiles, haciendo que cada vez los buenos lectores sean mejores y se distancien más de los malos lectores. Además, los buenos lectores aprenden más sobre el mundo y, por tanto, sería probable que su puntuación en el CI fuese más alta. Stanovich $(1986,1989)$ considera que es muy difícil que la "especificidad" de los déficits de los niños con DA se mantenga según el niño se va haciendo mayor, pues eso supondría que estos niños son inmunes a las consecuencias negativas de ser malos lectores. Además, según Sternberg y Grigorenko (2000), a los niños con dificultades en lectura se le suelen ofrecer textos de niveles bajos, es decir, a los niños que están en $5^{\circ}$ de Educación Primaria con un nivel de lectura de $3^{\circ}$ se le ofrecen lecturas de un nivel de $3^{\circ}$, lo que en su opinión es un grave error, pues el contenido y la complejidad del texto puede ser demasiado fácil y aburrido para ellos.

También Siegel y Himel (1998) observan cómo este "efecto Mateo" afecta al CI, y en particular al vocabulario, ya que las puntuaciones de los niños disléxicos mayores son significativamente más bajas que las de los niños disléxicos de menos edad. Sin embargo, las puntuaciones estandarizadas de los niños con problemas lectores en el test de Cubos permanecen relativamente constantes, lo cual es interpretado por los autores como un declive con el paso del tiempo de las habilidades relacionadas con el conocimiento adquirido mediante la lectura (Siegel, 1999). Ahora bien, debido a que con la edad van puntuando peor en algunas de las pruebas de los tests psicométricos, la discrepancia entre el CI y la competencia curricular se va haciendo menor, llevando a que tengan menos posibilidades de ser calificados como disléxicos en aquellos contextos escolares en los que se exige que la discrepancia CI-rendimiento sea significativa.

El estudio de Shaywitz, Holford y Holahan (1995) sobre los datos de los 21 niños con Dificultades en Lectura (DL) que participaron en el "Connecticut Longitudinal Study" desde Educación Infantil hasta Educación Secundaria encuentra un pequeño 
"efecto Mateo" sobre el CI, pero no sobre las habilidades lectoras. Por su parte, Scarborough y Parker (2003) llevaron a cabo un estudio longitudinal (de $2^{\circ}$ a $8^{\circ}$ ) con 57 niños con Dificultades de Aprendizaje de diverso tipo, evaluando la ejecución lectora, el CI y los problemas de conducta. Pudieron observar que el "efecto Mateo" no se producía en la mayoría de los niños, aunque entre los niños DAM las diferencias de CI respecto al grupo control se hacían mayores según iban pasando los cursos, además en este grupo también aumentaban los problemas de conducta.

Otro argumento en contra de la idea de que el CI no se ve afectado por las DA procede de la teoría de la "self-organization". Esta teoría sostiene que cuando un área cerebral se ve afectada por una lesión o disfunción (por ejemplo el lenguaje por lesión izquierda) se alteraría tanto la función controlada por esa área (el lenguaje) como la función que debería controlar el área que se ha tenido que encargar de las funciones alteradas (en este caso las habilidades viso-espaciales por implantación del lenguaje en el hemisferio derecho). Esto hace que no siempre se puedan observar las diferencias de CI entre la escala global de inteligencia y el rendimiento en una competencia curricular determinada (Zera, 2001).

Igualmente, en varios estudios (Dark y Bendow, 1990, 1991; Geary y Brown, 1991) se ha constatado que los niños con bajo CI obtienen rendimientos significativamente más bajos que los niños con CI en la media. Por ejemplo, Hoard, Geary y Hamson (1999) compararon a un grupo de 19 niños de $1^{\circ}$ con CI por debajo de 85 (obtenido en base a las puntuaciones de vocabulario y cubos del WISC) con el de un grupo de 43 niños de la misma edad con CI por encima de 85 constatando diferencias significativas en denominación de números, escritura de números, comparación de magnitudes, detección de errores de doble conteo y cálculo mental (p.e.: 9+9).

Por otra parte, conviene determinar el punto de corte que nos permita definir las dificultades de aprendizaje. Efectivamente, debido a que las habilidades humanas se distribuyen según la curva normal necesitamos establecer una puntuación que separe la normalidad de lo que no consideramos normal, pues sólo los casos extremos están claros. Muchos autores emplean como criterio para sus definiciones operativas de las DAM el rendimiento en pruebas estandarizadas de matemáticas por debajo del percentil 30 (Geary, Hoard y Hamson, 1999) o 35 (Geary, Hamson y Hoard, 2000; Hanich, Jordan, Kaplan y Dick, 2001; Jordan, Hanich y Kaplan, 2002, 2003). Pero en este caso el $30 \%$ ó $35 \%$ de la población presentarían dificultades de aprendizaje, sin embargo muchos de estos niños son falsos positivos, es decir, posteriormente presentan una evolución normal (Geary, 2003; Landerl, Bevan y Butterworth, 2004; Siegel, 1999), y además en la mayoría de las estadísticas se habla de que un $7 \%$ ó $10 \%$ de la población tiene Dificultades de Aprendizaje (Badian,1983; Bzufka, Hein y Neumaker, 2000; Gross-Tsur, Manor y Shalev, 1996; Kosc, 1974; Lyon, 1996; Ostad, 1998, 2002).
Otros autores emplean criterios más estrictos, como hacen Shalev, Manor y Gross-Tsur (1997) que seleccionan a los niños de $5^{\circ}$ que tienen 2 años de retraso. Igualmente Landerl et al. (2004) seleccionan aquellos alumnos que han señalado los profesores y que presentan una ejecución matemática por debajo de 3 desviaciones típicas de la media del grupo control.

También se están empezando a emplear definiciones de tipo dinámico, como son las de "respuesta a la intervención”. Según este modelo la identificación se produce en dos etapas, en la primera se establece el estatus de alumnos "con riesgo de dificultades" mediante la constatación de una ejecución académica más baja, usando para ello los criterios de corte que tradicionalmente se han propuesto (percentil 25 a 30, dependiendo de los casos). Tras lo cual se les somete a un programa de "intervención-valoración" durante un tiempo limitado. En el caso de los niños que no respondan de forma adecuada a la intervención se estima que tienen dificultades y que requieren el apoyo de Educación Especial de forma intensiva a largo plazo. Mediante este procedimiento se descarta que las dificultades se deban a pobre instrucción y a la vez se previene que estos niños mal atendidos se vayan retrasando en el sistema educativo (Berninger y Abbott, 1994; Carnine, 2003; Fuchs y Fuchs, 2002; Lyon et al., 2001; Scruggs y Mastropieri, 2002; Torgesen, 2001).

Teniendo en cuenta estas ideas, y para evitar la posible contaminación de falsos positivos, en el presente trabajos empleamos la determinación de "riesgo" del tutor, un criterio de bajo rendimiento restrictivo (por debajo de $1.50 \mathrm{DT}$ ) y la constatación de que las dificultades persisten a lo largo de un ciclo escolar (2 años).

El objetivo de este estudio es doble:

a) Contrastar si en los alumnos con Dificultades de Aprendizaje en Matemáticas (DAM) se produce el "efecto Mateo" en el CI, es decir, si la media de CI de los niños de menos edad es significativamente superior a la de los niños de más edad.

b) Contrastar el papel que juega el CI en relación con el rendimiento en matemáticas, o en otros términos, si el pobre rendimiento en matemáticas se asocia con bajas puntuaciones en CI.

Método

\section{Participantes}

Para la selección de la muestra se contactó con los tutores de 28 colegios que habían trabajado con los niños en el último curso de Educación Infantil y en $2^{\circ}$ de Educación Primaria y se les pidió que nos indicasen los alumnos de sus clases que podrían presentar "riesgo de sufrir dificultades de aprendizaje en matemáticas". Consideramos, al igual que Sternberg y Grigorenko (2000), que la opinión del tutor, en muchos casos, aporta información más útil que los tests psicométricos. 
Para determinar si realmente el alumno seleccionado por el tutor presentaba "Dificultades específicas de Aprendizaje en Matemáticas” (DAM) debía también cumplir las siguientes condiciones:

- Obtener en la prueba PRECUMAT (ver más adelante) una puntuación que se desviase al menos $1.50 \mathrm{DT}$ de la media obtenida por niños sin dificultades.

- Este retraso no puede imputarse a un nivel de inteligencia por debajo de la normalidad (debía ser superior a 80), padecer problemas emocionales graves, presentar algún tipo de déficit físico o sensorial, o no haber recibido una adecuada escolarización (Bermejo, Lago y Rodríguez, 1997; Blanco y Bermejo, 2004).

Los tutores consideraron que 18 niños de $1^{\circ}$ de Educación Primaria y 27 niños de $3^{\circ}$ de Educación Primaria eran alumnos de "riesgo". Además se seleccionaron otros dos grupos control de las mismas cohortes, con el fin de comparar la ejecución de estos niños DAM y los niños sin DAM. Las muestras control se obtuvieron aplicando las mismas cuotas observadas en el grupo experimental respecto a su edad, tipo de colegio (concertado o público) y género. La población total fue de 90 niños. Tras la valoración a lo largo del ciclo en el que estaban escolarizados y la aplicación de los criterios mencionados anteriormente la muestra se redujo a 71 niños, distribuidos tal y como recogemos en la tabla 1. Como puede observarse, el diseño utilizado es transversal, como lo son más del $90 \%$ de trabajos evolutivos que se publican, debido a las ventajas conocidas de este método.

Tabla 1. Distribución de participantes por muestras y ciclos.

\begin{tabular}{ccc}
\hline Grupos & Primer Ciclo & Segundo Ciclo \\
\hline SDAM & 17 & 22 \\
\hline DAM & 12 & 20
\end{tabular}

Nota: SDAM: niños sin dificultades de aprendizaje en matemáticas; DAM: niños con dificultades de aprendizaje en matemáticas.

\section{Procedimiento}

La evaluación de los alumnos se llevó a cabo al inicio del curso de forma individual en un aula habilitada para ese fin en las dependencias del centro educativo y dentro del horario escolar. Los alumnos de $1^{\circ}$ y $3^{\circ}$ fueron evaluados en matemáticas y mediante pruebas psicométricas de capacidades.

\section{Instrumentos}

La Prueba de Competencia Curricular de Primer Ciclo "PRECUMAT" ha sido elaborada por uno de los autores de este trabajo (Blanco, 1999) y adaptada al currículo de los distintos ciclos. Aquí empleamos la prueba de "Infantil" y la del "Primer Ciclo". Con ellas se puede obtener una puntuación global en matemáticas y puntuaciones parciales en: escritura y lectura de números, conteo, cálculo, problemas y hechos numéricos.
Con el fin de calcular el Cociente Intelectual se administraron las pruebas de "Semejanzas" y "Cuadrados" del WPPSI a los niños de $1^{\circ}$ de E.P. y la de "Semejanzas" y "Cubos" del WISC-R a los $3^{\circ}$ de E.P. A partir de estas puntuaciones se calculó el CI ponderado siguiendo las instrucciones de Groth-Marnat (2003) y aplicando las tablas de equivalencias de Sattler (1997).

\section{Resultados}

Con el fin de comprobar la primera de las hipótesis u objetivos planteados, comparamos primero los rendimientos de los niños DAM con el de los niños de la muestra control de la misma edad en las pruebas de CI. Los resultados de la t de Student muestran que los niños DAM tienen un rendimiento inferior al de los niños de la muestra control en las pruebas de CI de Whechsler, pero estas diferencias no son significativas en $1^{\circ}$, en cambio sí son significativas en $3^{\circ}$ (ver tabla 2 ).

Tabla 2. Comparación del CI en niños DAM y SDAM de $1^{\circ}$ y $3^{\circ}$ curso.

\begin{tabular}{cccccc}
\hline Cursos & & $M$ & $D T$ & $t$ (g.l.) & $p$ \\
\hline \multirow{2}{*}{ Primero } & DAM & 98.92 & 13.69 & & \\
& SDAM & 108.06 & 16.09 & $-1.60(27)$ & .112 \\
\multirow{2}{*}{ Tercero } & DAM & 87.50 & 10.72 & & \\
& SDAM & 107.14 & 13.56 & $-5.17(40)$ & .000 \\
\hline
\end{tabular}

Nota: SDAM: niños sin dificultades de aprendizaje en matemáticas; DAM: niños con dificultades de aprendizaje en matemáticas; CI: Cociente Intelectual.

Por otra parte, la diferencia entre los niños DAM de $1^{\circ}$ y de $3^{\circ}$ es estadísticamente significativa ( $t$ de Student) en las pruebas de CI, mientras que la diferencia de CI de los niños sin DAM pertenecientes a los dos grupos de edad no es significativa (ver tabla 3).

Tabla 3. Comparación del CI entre los niños DAM y SDAM.

\begin{tabular}{cccccc}
\hline Grupo & Curso & $M$ & $D T$ & $t$ (g.l.) & $p$ \\
\hline \multirow{2}{*}{ DAM } & $1^{\circ}$ & 98.92 & 13.69 & & \\
& $3^{\circ}$ & 87.50 & 10.72 & $-2.63(30)$ & .013 \\
\multirow{2}{*}{ SDAM } & $1^{\circ}$ & 108.06 & 16.09 & & \\
& $3^{\circ}$ & 107.14 & 13.56 & $-.19(37)$ & .847 \\
\hline
\end{tabular}

Nota: SDAM: niños sin dificultades de aprendizaje en matemáticas; DAM: niños con dificultades de aprendizaje en matemáticas; CI: Cociente Intelectual.

Podríamos pensar que el grupo de DAM de $1^{\circ}$ es distinto al de $3^{\circ}$, pero los datos sobre su competencia curricular en matemáticas nos hacen pensar que se trata del mismo tipo de alumnos, pues no se observan diferencias significativas ( $p=.05$, $t$ de Student) en la Competencia Curricular en $3^{\circ}$ entre los niños detectados en $1^{\circ}(M=293)$ y los detectados en $3^{\circ}(M=342)$. 
El segundo objetivo planteado pretende saber si la pobre ejecución en matemáticas de los niños DAM se podría atribuir a su bajo CI. Con este fin dividimos el grupo de niños sin dificultades (SDAM) en niños con CI superior a 100 y niños con CI inferior a 100, para posteriormente comparar la ejecución de estos dos grupos resultantes en la Prueba de Competencia Matemática con la obtenida por los niños DAM.

En primer lugar analizamos las diferencias en CI mediante un análisis de varianza (ANOVA) para un factor (DAM vs DAM con CI inferior a 100 vs. DAM con CI superior a 100) y la prueba $T 3$ de Dunnett de contraste a posteriori. De esta comparación se desprende que los grupos se diferencian de forma significativa $\left(F_{2.68}=99.1, p=.000\right)$, pero este resultado se debe principalmente a las diferencias observadas entre los dos grupos de niños sin dificultades, como podemos ver en la tabla 4. El CI de los niños DAM era ligeramente superior al de los niños SDAM "con CI inferior a 100", pero la diferencia entre estos dos grupos no resulta significativa (tabla 4).

En cuanto a la Competencia Matemática evaluada a inicios de $3^{\circ}$ mediante un contraste ANOVA similar se observó que las diferencias entre los grupos resultaron significativas $\left(F_{2,68}=\right.$ $86.66, p=.000)$. En efecto, en la tabla 5 podemos observar que los niños SDAM con "CI superior a 100" superan a los otros dos grupos, especialmente a los DAM, y que el grupo SDAM con "CI inferior a 100" es significativamente superior al grupo de niños DAM.

Tabla 4. Contrastes post hoc del CI con la T3 de Dunnett.

\begin{tabular}{cccccc}
\hline \multicolumn{1}{c}{ Grupos } & $\begin{array}{c}\text { Diferencias } \\
\text { entre medias }\end{array}$ & $\begin{array}{c}\text { Error } \\
\text { estándar }\end{array}$ & $p$ & \multicolumn{2}{c}{ IC al 95\% } \\
& & & & \multicolumn{2}{c}{ Límite inf. Límite sup. } \\
\hline SDAM, CI $>100-$ SDAM, CI $<100$ & 25.0926 & 2.69991 & .000 & 18.2519 & 31.9333 \\
SDAM CI $<100-$ DAM & -1.6146 & 3.05843 & .934 & -9.2555 & 6.0263 \\
DAM - SDAM CI $>100$ & $-23 . .4780$ & 2.90976 & .000 & -30.6328 & -16.3232 \\
\hline
\end{tabular}

Nota: SDAM: niños sin dificultades de aprendizaje en matemáticas; DAM: niños con dificultades de aprendizaje en matemáticas; CI: Cociente Intelectual.

Tabla 5. Contrastes post hoc de la ejecución en matemáticas en $3^{\circ}$ de Primaria con la T3 de Dunnett.

\begin{tabular}{lcccccc}
\hline \multicolumn{1}{c}{ Grupos } & $\begin{array}{c}\text { Diferencias } \\
\text { entre medias }\end{array}$ & $\begin{array}{c}\text { Error } \\
\text { estándar }\end{array}$ & $p$ & \multicolumn{2}{c}{ IC al 95\% } \\
& & & & Límite inf. Límite sup. \\
\hline SDAM, CI $>100-$ SDAM, CI $<100$ & 53.6615 & 24.06427 & .104 & -8.6442 & 115.9672 \\
SDAM CI $<100-$ DAM & 216.5209 & 26.31945 & .000 & 149.6997 & 283.3422 \\
DAM - SDAM CI $>100$ & -270.1824 & 21.25217 & .000 & -322.4428 & -270.9220 \\
\hline
\end{tabular}

Nota: SDAM: niños sin dificultades de aprendizaje en matemáticas; DAM: niños con dificultades de aprendizaje en matemáticas; CI: Cociente Intelectual.
Discusión

Como en estudios previos (Klinge et al., 1977; Torgesen y Dice, 1980; Stanovich, 1986) los niños DAM de nuestro trabajo tienen un CI inferior al de sus compañeros. Aunque tal diferencia es pequeña al inicio de la escolaridad, los niños DAM en $1^{\circ}$ presentan un CI $(M=98.92)$ similar al de los niños sin DAM $(M=108.06)$, mientras que los niños DAM en $3^{\circ}$ obtienen puntuaciones $\mathrm{CI}(M=87.50)$ significativamente inferiores a la de los niños de su edad sin dificultades $(M=107.14)$ y a la de los niños DAM de $1^{\circ}$. Estas diferencias en el rendimiento en las pruebas CI no se observan entre los dos cursos de niños sin dificultades. Tal caída de puntuaciones se podría atribuir a lo que Stanovich (1986) ha bautizado como el "efecto Mateo", de modo que las dificultades de aprendizaje privan a los alumnos de unas experiencias escolares que influyen en la ejecución de las pruebas de CI. Este efecto se ha evidenciado en estudios previos con niños con dificultades en lectura (Shaywitz et al., 1995; Siegel y Himel, 1998) y en niños con DAM (Scarborough y Parker, 2003).

Por otro lado, podemos afirmar que aunque en general los niños DAM tienen un CI más bajo que los niños sin DAM, no se pueden achacar sus dificultades en matemáticas a esta circunstancia, pues el rendimiento matemático de los DAM $(M=323.48)$ es significativamente más pobre que el de niños SDAM con un CI similar $(M=540)$. Igualmente, el grupo de niños control con "CI inferior a 100 " obtiene puntuaciones inferiores en competencia matemática a las alcanzadas por los niños con "CI superior a 100" $(M=593.66)$, pero éstas no son significativas y no hace que sean identificados por los profesores como niños con dificultades, así como que no alcancen una competencia matemática aceptable, promocionando de un curso a otro sin problemas. Estos últimos resultados no confirman los obtenidos por el equipo de Geary (Geary y Brown, 1991; Hoard et al., 1999), ya que estos autores sí encontraban diferencias entre los niños en función del CI. Pero estas discrepancias podrían deberse a que las características de los niños eran distintas en ambos trabajos. Efectivamente, en el estudio de Geary el grupo con CI alto eran niños con "talento académico" y en el segundo grupo los niños con CI bajo eran niños "límites" (CI inferior a 85). Es decir, en estos estudios se seleccionan niños posicionados más lejos de la media que en nuestro trabajo.

Concluimos subrayando la necesidad de detectar a los niños con dificultades lo más precozmente posible, ya que sólo a edades tempranas evitamos confundir el diagnóstico debido a la "caída" de las puntuaciones de CI, pudiendo constatar más fácilmente la especificidad de las DAM. Más tarde puede que no sea posible. Por otro lado, esta detección precoz no sólo permitirá intervenir en el área de su dificultad, sino también a no descuidar aquellas habilidades en las que el niño es más competente, ya que si se trabajan adecuadamente le ayudarán en el futuro, al menos, a tener una autoestima más positiva. 
Referencias

Badian, N.A. (1983). Dyscalculia and nonverbal disorders of learning. En H.R. Myklebust (Ed.), Progress in learning disabilities (vol.5, pp. 235-263). Nueva York: Grune y Stratton.

Bermejo, V., Lago, Ma O. y Rodríguez, P. (1997). Dificultades de aprendizaje de las matemáticas. En J. N. García (Ed.), Instrucción, aprendizaje y dificultades (pp. 383-395). Barcelona: EUB.

Berninger, V.W. y Abbott, R.D. (1994). Redefining learning disabilities: Moving beyond aptitude-achievement discrepancies to failure to respond to validated treatment protocols. En G.R. Lyon (Ed.), Frames of reference for the assessment of learning disabilities: New views on measurement issues (pp. 163-183). Baltimore, Maryland: Paulh Brookes Publishing.

Blanco, M. (1999). Desarrollo de un instrumento de evaluación, diagnóstico y orientación curricular del área de Matemáticas en los primeros años de escolaridad: Prueba evolutivo-curricular de matemáticas de Tordesillas (PRECUMAT). Premios nacionales a la innovación educativa 1999, documento no publicado.

Blanco, M. y Bermejo, V. (2004). Dificultades de aprendizaje en matemáticas. En V. Bermejo (Coord.), Cómo enseñar matemáticas para aprender mejor (pp. 215-238). Madrid: CCS.

Brody, L.E. y Mills, C.J. (1993). Gifted children with learning disabilities: a review of the Issues. Journal of Learning Disabilities, 30, 283-296.

Bzufka, M.W.; Hein, J. y Neumaker, K.J. (2000). Neuropsichological differentiation of subnormal arithmetic abilities in children. European Child and Adolescent Psychiatry, 9, 65-76.

Carnine, D. (2003). IDEA: Focusing on Improving Results for Children with Disabilities. Commitee on Education and the Workforce. www.house.gov/search97cgi.

Dark, V.J. y Benbow, C.P. (1991). Differential enhancement of of working memory with mathematical versus verbal precocity. Journal of Educational Psychology, 83, 48-60.

Fuchs, L.S. y Fuchs, D. (2002). Progress monitoring, accountability, and LD identification. Testimony to the Presidents Commission on Excellence in Special Education. Extraído del sitio Web: http://www.cecdr.org/content/index.cfm? contentID $=28 \&$ Order $=$ ASC

García, A. I. y Jiménez, J. E. (2002). Resolución de problemas verbales aritméticos en niños con dificultades de aprendizaje. Cognitiva. 12, 153-170.

Geary, D. C. (1994). Children's Mathematical Development, Research and Practical Applications. Washington, DC: American Psychological Association.

Geary, D. C. (2003). Learning disabilities in arithmetic: Problem-solving differences and cognitive deficits. En H.L.
Swanson, K.R. Harris y S. Graham (Eds.), Handbook of Learning Disabilities (pp. 119-212). Nueva York: The Guilford Press.

Geary, D.C. y Brown, S.C. (1991). Cognitive addition: strategy choice and speed-of-processing differences in gifted, normal and mathematically disabled children. Developmental Psychology, 27, 398-406.

Geary, D.C.; Hamson, C.O. y Hoard, M.K. (2000). Numerical and arithmetical cognition: a longitudinal study of process and concept deficits in children with learning disability. Journal of Experimental Child Psychology, 77, 236-263.

Geary, D.C.; Hoard, C.O. y Hamson M.K. (1999). Numerical and arithmetical cognition: patterns of functions and deficits in children at risk for a mathematical disability. Journal of Experimental Child Psychology, 74, 213-239.

Groth-Marnat, G. (2001). The Wechsler intelligence scales. En A. Kaufman y N.L. Kaufman (Eds.), Specific Learning disabilities and difficulties in children and adolescent. Psychological assessment and evaluation (pp. 29-51). Cambridge: Cambridge University Press.

Groth-Marnat, G. (2003). Handbook of Psychological Assessment. Hobohen, Nueva Jersey: John Wiley and Sons.

Hanich, L.B.; Jordan, N.C.; Kaplan, D. y Dick, J. (2001). Performance across different areas of mathematical cognition in children with learning difficulties. Journal of Educational Psychology, 93, 615-626.

Hoard, M.K.; Geary, D.C. y Hamson, C.O. (1999). Numerical and Arithmetical Cognition: Performance of Low- and Average-IQ Children. Mathematical Cognition, 5, 65-91.

Jordan, N.C.; Kaplan, D. y Hanich, L.B. (2002). Achievement growth in children with learning difficulties in mathematics: Findings of a two-year longitudinal study. Journal of Educational Psychology, 94, 586-597.

Jordan, N. C.; Hanich, L. B. y Kaplan, D. (2003). A longitudinal study of mathematical competencies in children with specific mathematics difficulties versus children with comorbid mathematics and reading difficulties. Child Developmental, $74,834-850$.

Keogh, B. (1990). Definitional assumptions and research issues. En H.L. Swanson y B. Keogh. (Eds.), Learning disabilities: theoretical and research issues (pp. 13-19). Hillsdale, Nueva Jersey: Lawrence Erlbaum.

Klinge, V.; Rennick, P.; Lennox, K. y Hart, Z. (1977). A matched-subjeCt comparison of underachievers with normals on intellectural, bahevioral, and emotional variables. Journal of Abnormal Child Psychology, 5, 61-68.

Kosc, L. (1974). Developmental dyscalculia. Journal of Learning Disabilities, 7, 164-177.

Landerl, K., Bevan, A y Butterworth, B., (2004). Developmental dyscalculia and basic numerical capacities: a study of 8-9 years old students. Cognition, 93, 99-125.

Lyon, G.R. (1996). Learning disabilities. Special education for students with disabilities. The Future of Children, 6, 
54-76. Extraído del sitio web: http://www.ldonline.org/ ld_indepth/general_info/future_children.html

Lyon, G. R.; Fletcher, J.M.; Shaywitz, S.E., Shaywitz, B.A.; Torgesen, J.K.; Wood, F.B., Shulde, A. y Olson, R. (2001). Rethinking learning disabilities. En C. E. Finn, A. J. Rotherham y C. R. Hokanson (Ed.), Rethinking special education for a new century (pp. 259-287). Washington: The Thomas B. Fordham Foundation.

Ostad, S. A. (1998). Developmental differences in solving simple arithmetic word problems and simple number-fact problems: A comparison of mathematically normal and mathematically disabled children. Mathematical Cognition, 4, 1-19.

Ostad, S. A. (2002). Mathematical difficulties: Aspects of learner characteristics in developmental perspective. Neuron, 40, 847-858. Extraído del sitio web: http://folk.vio.no/snorreo/ paper1.doc

Sattler, J.M. (1997). Evaluación de la inteligencia infantil. Santafé de Bogotá, Méjico: Manual Moderno (Orig. 1974).

Shalev, R.S., Manor, O. y Gross-Tsur, V. (1997). Neuropsychological aspects of developmental dyscalculia. Mathematical Cognition, 3, 105-120.

Shaywitz, B.; Holford, T.R. y Holahan, J.M. (1995). A Matthew Effect for IQ but Not for Reading: Results from a Longitudinal Study. Reading Research Quarterly, 30, 894-906.

Siegel, L.S. (1990) IQ and learning disabilities: R.I.P.. En H.L. Swanson y B. Keogh (Eds.), Learning disabilities: theoretical and research issues (pp.111-194). Hillsdale, Nueva Jersey: Lawrence Erlbaum.

Siegel, L.S. (1999). Issues in the definition and diagnosis of learning disabilities: a perspective on Guckenberger v. Boston University. Journal of Learning Disabilities, 32, 304-319.
Siegel, L.S. y Himel, N. (1998). Socioeconomic status, age and the classification of dyslexics and poor readers: the dangers of using IQ scores in the definition of reading disability. Dyslexia, 4, 90-104.

Scruggs, T. E. y Mastropieri, M. A. (2002). On babies and bathwater: addressing the problems of identification of learning disabilities. Learning Disabilities Quarterly, 25, 155-168.

Stanovich, K.E. (1986). Matthew effects in reading: Some consequences of individual differences in the acquisition of literacy. Reading Research Quarterly, 21, 360-407

Stanovich, K.E. (1989). Has the Learning Field Lost its Intelligence? Journal of Learning Disabilities, 22, 487-492.

Sternberg, R. J. y Grigorenko, E.L. (2000). Our labeled children. What Every Parent and Teacher Needs to Know About Learning Disabilities. Cambridge, Massachusetts: Perseus Publishing Group.

Torgesen, J. (2001). Empirical and theorical support for direct diagnostic of learning disabilities by assessment of intrinsic processing weaknesses. En National Research Center of Learning Disabilities, Learning Disabilities summit: Building a foundation for the White Paper. Extraído del sitio Web: www.nrcld.org/html/information/articles/ ldsummit/

Torgesen, J. y Dice, C. (1980). Characteristics of research in learning disabilities. Journal of Learning Disabilities, 13, 531-535.

Fecha de recepción: 3 de Julio de 2009 Fecha de aceptación: 12 de noviembre de 2009 